\title{
Energy exponents of avalanches and Hausdorff dimensions of collapse patterns
}

\author{
Blai Casals $\odot$ and Ekhard K. H. Salje $\odot$ \\ Department of Earth Sciences, University of Cambridge, Downing Street, Cambridge CB23EQ, United Kingdom
}

(Received 19 June 2021; revised 23 September 2021; accepted 5 November 2021; published 29 November 2021)

\begin{abstract}
A simple numerical model to simulate athermal avalanches is presented. The model is inspired by the "porous collapse" process where the compression of porous materials generates collapse cascades, leading to power law distributed avalanches. The energy $(E)$, amplitude $\left(A_{\max }\right)$, and size $(S)$ exponents are derived by computer simulation in two approximations. Time-dependent "jerk" spectra are calculated in a single avalanche model where each avalanche is simulated separately from other avalanches. The average avalanche profile is parabolic, the scaling between energy and amplitude follows $E \sim A_{\max }^{2}$, and the energy exponent is $\varepsilon=1.33$. Adding a general noise term in a continuous event model generates infinite avalanche sequences which allow the evaluation of waiting time distributions and pattern formation. We find the validity of the Omori law and the same exponents as in the single avalanche model. We then add spatial correlations by stipulating the ratio $G / N$ between growth processes $G$ (linked to a previous event location) and nucleation processes $N$ (with new, randomly chosen nucleation sites). We found, in good approximation, a power law correlation between the energy exponent $\varepsilon$ and the Hausdorff dimension $H_{\mathrm{D}}$ of the resulting collapse pattern $H_{\mathrm{D}}-1 \sim \varepsilon^{-3}$. The evolving patterns depend strongly on $G / N$ with the distribution of collapse sites equally power law distributed. Its exponent $\varepsilon_{\text {topo }}$ would be linked to the dynamical exponent $\varepsilon$ if each collapse carried an energy equivalent to the size of the collapse. A complex correlation between $\varepsilon, \varepsilon_{\text {topo }}$, and $H_{\mathrm{D}}$ emerges, depending strongly on the relative occupancy of the collapse sites in the simulation box.
\end{abstract}

DOI: 10.1103/PhysRevE.104.054138

\section{INTRODUCTION}

Systematic investigations of avalanche phenomena began when geophysicists tried to understand the origin of the Gutenberg-Richter law of earthquakes [1,2]. In particular, the enigma of aftershocks after large quakes and, even more importantly, the attempted discovery of preshocks, which could serve as early warning signs of major earthquakes, led to pioneering developments of mathematical models [3]. Models, such as trigger models and the epidemic type aftershock sequence (ETAS) models [3], partially explained the dynamics of earthquakes but, unfortunately, did not reach the main goal of earthquake prediction [4]. These models, the stick-slip model [5-7], the fiber bundle model [8-11], and simple Ising models [12] form the backbone for our current understanding of avalanche processes with several more detailed models developed for specific purposes. Avalanches were also associated with Barkhausen noise in magnets and martensitic noise in metals and alloys [13-15]. Notable contributions were made on the pinning-depinning model [16-18] and in fields such as sheared granular matter [19-22], imbibition [23,24], and rainfall $[25,26]$. Many other specific fields are explored in terms of avalanche research and further expansions of these activities are expected in coming years.

Over the last decade, another field of application in materials sciences developed. Avalanches were already observed in ceramics, dislocation movements, ferroelastic and ferroelectric materials during switching and phase transitions, and during the collapse of porous materials under stress.
These applications modified some of our perceptions of avalanches $[10,27,28]$. While the universality of avalanche behavior is often greatly appreciated, it increasingly turned into a curse in materials sciences and neuromorphic computing [29]. If all avalanches were similar with restricted fixed-point behavior, then all materials of large classes of materials will behave dynamically identically $[30,31]$. The key question now is what process or processes cause the avalanches and how can they be influenced by changing materials properties. The question is no longer whether avalanches exist. Often, not much can be learned about the inner mechanics of ferroic switching or the ways in which porous materials collapse from experimentally analyzing avalanche movements. This view turned out to be, at least partially, too negative. Several observations already point to specific processes starting from the evolution of energy exponents and the superposition of avalanches, and process-dependent prefactors of scaling laws $[32,33]$.

One key observation of physical processes, such as the porous collapse or the movement of ferroic domains, is that they form specific dynamic patterns during the evolution of the avalanche. The unique aspect is that geometrical patterns can be observed, e.g., in optical microscopy, and their evolution can be measured. It was found that the changes of pattern are often fractal and that direct anticorrelations between the Hausdorff dimension of the pattern evolution and the avalanche scaling of their energies or amplitudes were observed [34]. So far, these observations are empirical and do not distinguish between exponents of dynamical processes and 
topological configurations, but it is anticipated that with the advent of more experiments with much higher time and space resolution, the detailed characterization of the pattern formation process will advance further. To provide a framework for such studies, we propose a simple and intuitive avalanche model which allows us to simulate the pattern evolution very easily. The model is motivated by the observation of the porous collapse in the model material Vycor [35-38] where avalanches are experimentally observed over seven decades with virtually no experimental fingerprints of cutoff effects. This porous material consists of $\mathrm{SiO}_{2}$ clusters which surround cavities. The diameter of the cavities varies greatly and the solid matrix is amorphous in diffraction experiments; i.e., it forms a glass. The collapse mechanism is understood to be related to the local collapse of a cavity which reduces the local specific volume and triggers the collapse of other cavities via the emitted strain waves [39]. The stochastic nature of the distribution of cavities and their strain interactions gives rise to the formation of avalanches. This behavior is then compared with the domain movements of ferroelastic and ferroelectric domains in $\mathrm{BaTiO}_{3}$ where the same avalanche parameters were found. Indeed, it seems generally assumed that the pinning-depinning universality class covers both the porous collapse and the movement of ferroelectric domains under an electric field although an atomistic picture of the collapse mechanism (unlike the domain movement) is still missing. However, we anticipate this model can be used in analogy for other crackling systems such as crack propagation, sheared amorphous materials, collapsing sand piles, or domain wall motion.

Avalanches in $\mathrm{BaTiO}_{3}$ have the advantage that the Hausdorff dimension of the emerging domain patterns can be evaluated directly by optical observations [34]. Our model is designed to answer the question: What pattern evolution is expected during porous collapse and what correlations exist between dynamic avalanche parameters (e.g., the exponents of power law distributions), the collapse pattern, and the Hausdorff dimension.

\section{COLLAPSE MODEL WITH A SINGLE AVALANCHE EVENT}

A temporal avalanche profile is defined by the number of collapsed sites per unit of time. Experimentally, the avalanches are usually measured by acoustic emission or by the time evolution of a certain magnitude such as the birefringence or the spontaneous polarization [40-42]. In this case, distinguishing between avalanches is not a trivial task. This problem is commonly overcome by using a threshold for observables like amplitudes and energies of the avalanches to split and label individual events. However, some features can be undistinguishable, for instance, the time overlap between two or more independent avalanches or the use of an overestimated threshold could split a single avalanche. Our first goal is to generate individual avalanche profiles. We consider that the number of collapsed sites per unit of time $N(t)$ is a consequence of all previous collapses $N(t-1)$. We model this triggering by a factor $f(t)$, which is described by a probability distribution function. Thus, the expression for a single avalanche profile $N(t)$ can be written as

$$
N(t)=f(t) N(t-1)
$$

The initial condition is the collapse of a single, randomly chosen site, $N(0)=1$. The avalanche duration is determined by the time span until $N(t)$ reaches zero $N(t)=0$, which terminates the avalanche. We refer to this model as the "single event model." In order to obtain a sufficiently large number of avalanches for a statistical analysis, the model is run many times from a new random initial condition; each run lasts until the avalanche stops when $N(t)$ reaches zero. In this model, the number of sites involved in an avalanche is always small compared with the total number of sites so that there is no danger of unwanted finite-size effects of the model.

We explored the distribution of avalanche energies, amplitudes, and sizes by running numerical simulations using Eq. (1) with several probability functions $f(t)$ to generate statistically relevant datasets for single avalanches. We found that a Gaussian probability function $f(t)$ with a width $w_{f}=\sqrt{2}$ leads to avalanches with power law distributed avalanche energies (Fig. 1) with a power law exponent of $\varepsilon=1.33$, which is the same as in mean field theories [40]. We use the same $w_{f}=\sqrt{2}$ for all simulations. However, the power law exponent can be modified by changing the width $w_{f}$ of the distribution function $f(t)$. Examples are given in the Supplemental Material ([43], Fig. S1). Some typical avalanche profiles $N(t)$ are displayed in Fig. 1(a). We defined the maximum amplitude of an avalanche profile as $A_{\max }=$ $\max (|N(t)|)$, their size as $S=\int_{0}^{D}|N(t)| d t$, and their energy as $E=\int_{0}^{D} N(t)^{2} d t$ where $D$ is the event duration. The avalanche profiles are normalized by the maximum amplitude for each avalanche in Fig. 1. The quantities $E, A_{\max }$, and $S$ follow power law distributions with exponents $\varepsilon=$ $1.33, \tau^{\prime}=1.66$, and $\tau=1.66$, respectively. These distributions are analyzed by a logarithmic binning [Fig. 1(b)] and with the maximum-likelihood algorithm [Fig. 1(c)] that shows a well-defined maximum-likelihood exponent (MLE) over more than six decades in energy. The correlations between these quantities are equally power law distributed with exponents defined as $E \sim A_{\max }^{x}, S \sim A_{\max }^{\gamma}, A_{\max } \sim D^{\chi}$ [Figs. 1(d)-1(f)]. The close adherence to the power law of the first two correlations and the much greater data scatter of the last correlation are very similar to experimental observations [44-47]. The avalanche shape, obtained by averaging profiles normalized by its duration for different duration ranges, exhibits a slightly asymmetric parabola as expected for avalanche systems [Fig. 1(g)] [48-50].

\section{CONTINUOUS EVENTS MODEL}

Several key features of the avalanche statistics, such as the distributions of activities, interevent times, and main and aftershock relations, cannot be explored by the single event model because each avalanche is simulated individually with a new initial condition when an avalanche terminates. In the next step we construct an extended model, which allows us to explore the appearance of events in time. We refer to this model as the "continuous events model." To generate events as a function of time we add a "noise function" $g(t)$ to Eq. (1). The noise is represented by a random number with 
(a)

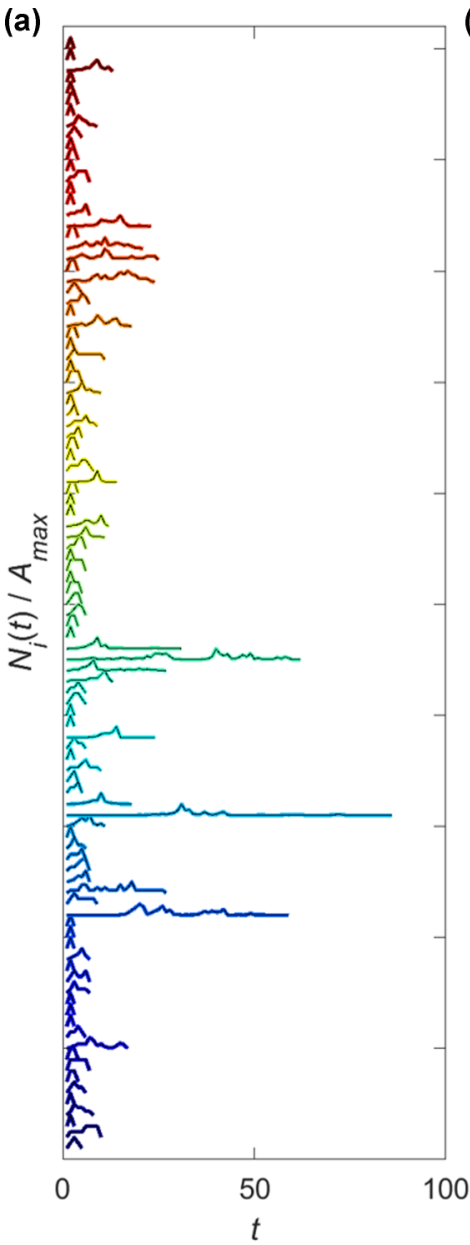

(b)

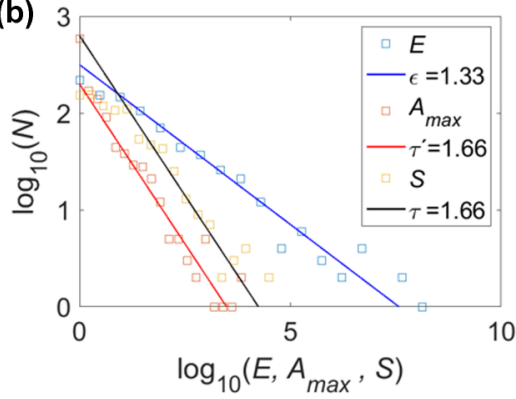

(d)
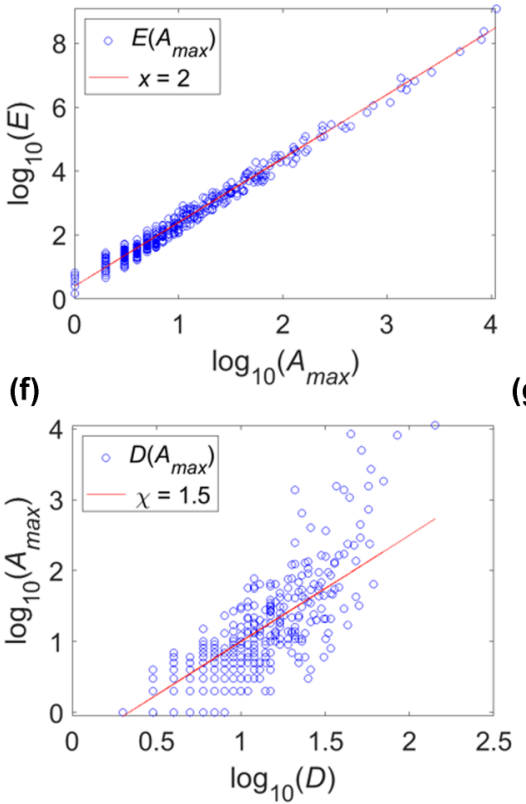

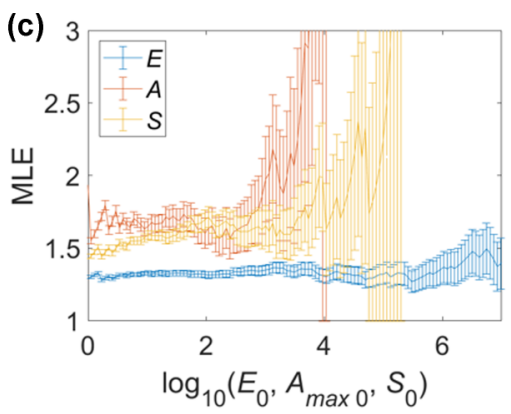

(e)

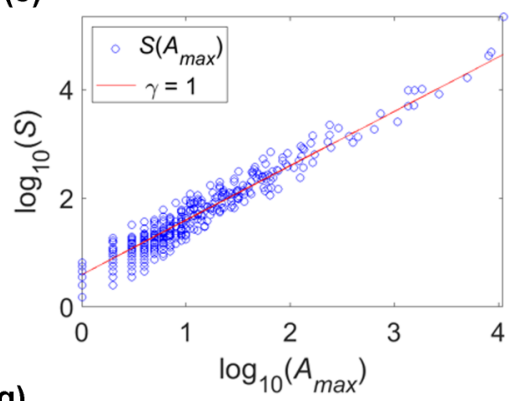

(g)

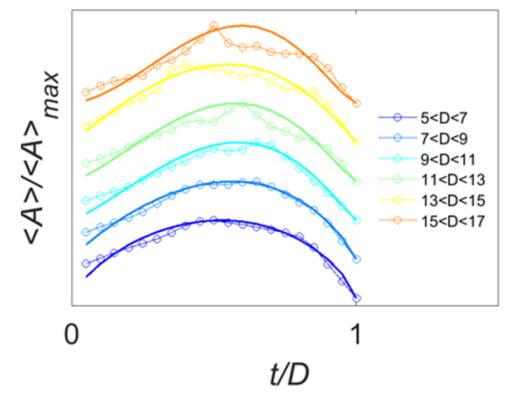

FIG. 1. Avalanche statistics from the single event model. (a) Example of 100 avalanche profiles, each generated by the single event model. (b) Distribution of the energies $E$, amplitudes $A$, and sizes $S$ for 1200 events showing exponents $\varepsilon=1.33 \pm 0.05, \tau^{\prime}=1.66 \pm 0.10$, and $\tau=$ $1.66 \pm 0.10$. (c) Maximum-likelihood exponent of the power laws for $E, A$, and $S$ confirming the exponents. Scaling relations $E(A)$ (d), $S(A)$ (e), and $A(D)$ (f). Averaged avalanche profile with normalized duration of profiles for different duration ranges (from five to 17 time steps). The plots are shifted upwards for better comparison. The avalanche shapes are fitted with the scaling function proposed in [49]. A larger dataset is needed to compute the avalanche shape for larger durations.

a flat probability distribution between 0 and 1 . It introduces a random noise that can trigger a new avalanche even when $f(t) N(t-1)=0$. The initial condition is now irrelevant, and large datasets are obtained by computing $N(t)$ as

$$
N(t)=f(t) N(t-1)+g(t) .
$$

The physical scenario for this model is for collapse avalanches in which thermal noise can initiate avalanches even when the external driving forces, such as the sample compression, are constant. Even more common is the effect of long-term relaxations in the system. After avalanches have released many of the internal strains, the final state relaxes over time periods which are long compared with the avalanche durations. These relaxations generate local stress points, which initiate subsequent avalanches, such as aftershocks [51].

The time evolution of the collapsed sites after $2 \times 10^{5}$ time steps is shown in Fig. 2(a). In order to split $N(t)$ into individual avalanche events, we have applied a threshold as is commonly done in experimental studies. We used a threshold condition that more than two collapsing sites are needed to define an avalanche. When an avalanche terminates, the term $g(t)$ will always restart a new avalanche. With the parameters chosen for $g(t)$, the individual avalanche profiles are close to the single event model. The probability distributions for the totality of all avalanches in the continuous events model exhibit power law behavior, with exponents $\tau=1.67$ and $\varepsilon=1.33$, that extends over ten decades [Fig. 2(b)]. The maximum-likelihood analysis is shown in the Supplemental Material (Fig. S2 [43]) confirming the found exponents. The scaling relations $E \sim A_{\max }^{x}, S \sim A_{\max }^{\gamma}, A_{\max } \sim D^{\chi}$ are found to be the same as in the single event model (Supplemental Material, Fig. S2 [43]).

We can now evaluate time-dependent quantities such as the interevent times (or waiting times) as the time interval between two avalanches. Experimentally, the rate of aftershocks $\left(r_{\mathrm{as}}\right)$ is quantified by the Omori law [52] which describes the statistical behavior of aftershocks (ASs). The main shocks are defined as shocks that belong to a certain energy range; then an aftershock is a smaller signal that occurs after the previous main shock. If an aftershock is larger than the main shock, the AS sequence is terminated, and this large shock is counted as a new main shock inside its appropriate energy range of main 

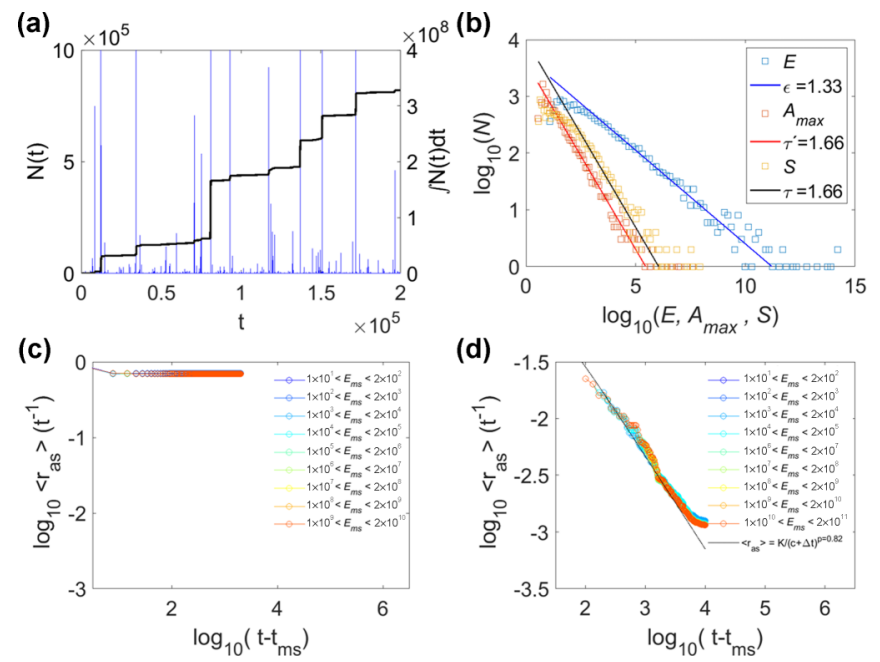

FIG. 2. Avalanche statistics from the continuous model. (a) $N_{s}(t)$ is the continuous avalanche sequence (the jerk spectrum) in blue. The black curve shows the accumulated collapsed sites as a function of time. (b) Power law distribution of the $E, A_{\max }$, and $S$ with the exponents $\varepsilon=1.33 \pm 0.05, \tau^{\prime}=1.66 \pm 0.10$, and $\tau=1.66 \pm 0.10$, respectively. (c) The rate of aftershocks $\left\langle r_{\text {as }}\right\rangle$ as a function of the time lapse $t-t_{\mathrm{ms}}$ after the end of the main shock $t_{\mathrm{ms}}$ for a stochastic function $g(t)$. No time correlations occur. (d) A typical Omori's curve is generated when $g(t)$ activates avalanches after a delay time which is randomly selected from a half-Gaussian distribution $\sim \exp (t / 2000)^{2}$ with $p=0.82$.

shocks. Omori's law states that the number of ASs decays as a power law after each main shock (MS) with a frequency of $r(t)=K(t+c)^{-p}$. For purely stochastic $g(t)$ there are no time correlations and $p=0$. Figure 2(c) shows the Omori plot of the probability of an aftershock as a function of the time interval between the end of the main shock and the onset of the aftershock for various ranges of main shock energies $E_{\mathrm{ms}}$ $[35,46,53]$. We now introduce a simple time correlation by stipulating that $g(t)$ is zero during an avalanche and remains zero after the end of the avalanche for a time span defined by a random number chosen from the positive branch of a Gaussian curve with a half width of 2000 time steps. The decay of the Gaussian function ensures that aftershocks are more likely to be close to the main shock and introduces an Omori-Utsu law [Fig. 2(d)] with $p=0.82$. We have assessed Omori's law for different Gaussian widths $w_{f}$ of $f(t)$ which give similar scaling independently from the power law exponents (Supplemental Material, Fig. S3 [43]).

\section{SPATIAL DISTRIBUTION OF THE SINGLE AND CONTINUOUS EVENTS MODEL}

We now explore the topological characteristics of pattern formation generated by the collapsed sites. Following the percolation cluster methods [54-56], we consider a matrix of pixels ( $\sim$ one frame) where one pixel is the smallest unit of collapse. Sites are denoted 0 when intact and 1 when collapsed, and remains as 1 even after further hits. Initially (at $t=0$ ) all pixels are in the 0 state. The pattern is contained within the simulation box of potential collapse sites. First, we use the single event model where $N(t)$ is the number

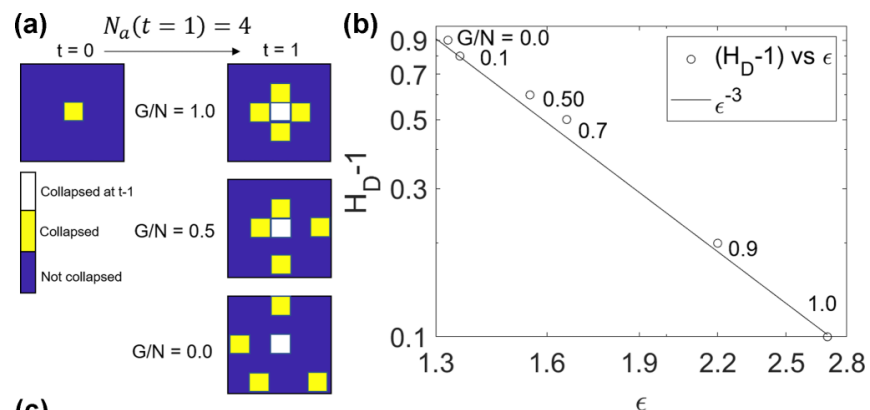

(c)
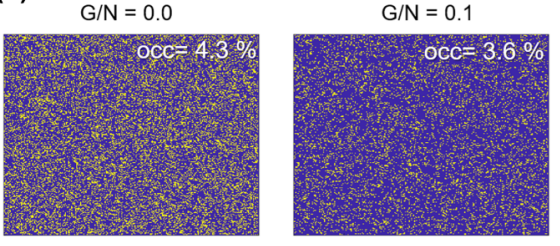

$\mathrm{G} / \mathrm{N}=0.5$
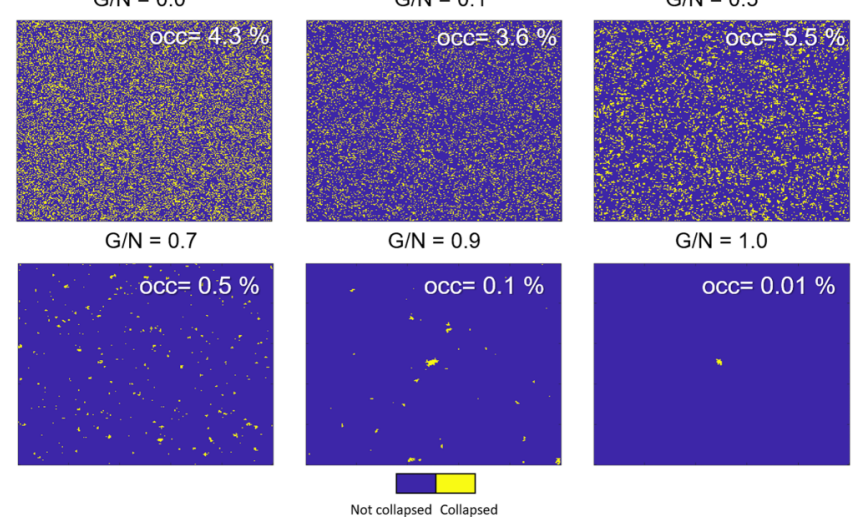

$\mathrm{G} / \mathrm{N}=1.0$

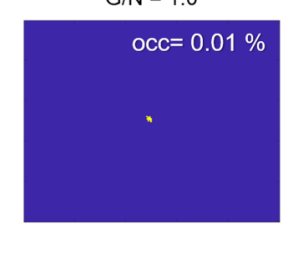

FIG. 3. Spatial distribution for the single event model. (a) Scheme of the spatial distribution as a function of the $G / N$ percentage. (b) The Hausdorff dimension $H_{\mathrm{D}}$ scales as a function of the energy exponent $\varepsilon$ for different $G / N$ percentage as $H_{\mathrm{D}}-1 \sim \varepsilon^{-3}$. The error for $\varepsilon$ and $H_{\mathrm{D}}$ is \pm 0.05 . (c) Examples of the largest event that occurred for different $G / N$ percentages. Images of the frame showing all collapsed areas after a single event.

of collapses to be distributed. As overlap between collapses occurs, we distinguish between attempted $N_{a}(t)$ and successful collapses $N_{s}(t)$. The attempted collapses $N_{a}(t)$ are again triggered by the previous successful collapses $N_{s}(t-1)$ as in the previous models. The single event model, including overlap, is written as

$$
N_{a}(t)=f(t) N_{s}(t-1) .
$$

Each event starts with an empty frame and ends when $N_{a}(t)=0$. To distribute $N_{a}(t)$ at each time step, we distinguish between sites which represent either "growth" of a pattern, namely, when the new collapsed site is attached to a previously collapsed site, or "nucleation" when this is not the case. The split between $N_{a}(t)$ sites in nucleation $N$ sites and growth sites $G$ uses the probability parameter $G / N$ as shown schematically in Fig. 3(a). The collapsed sites have no inherent spreading. The growth (given by the $G / N$ factor) stems from the spatial arrangement of the new attempted collapses. When $G / N=0$, all $N_{a}(t)$ are distributed by "nucleation" and are placed randomly in space, which includes places around the previous collapse sites. In the other extreme case, when $G / N=1, N_{a}(t)$ is distributed only by "growth" and the collapses are randomly distributed along the perimeters of previously collapsed areas. As an intermediate example, if $G / N=0.5,50 \%$ of $N_{a}(t)$ will be distributed by growth and $50 \%$ by nucleation. We have generated six datasets for $G / N=0,0.1,0.5,0.7,0.9$, and 1 and analyzed the power 
law exponent for all cases. In addition, the topological properties of the collapsed areas were analyzed by computing the Hausdorff dimension, $H_{\mathrm{D}}$, which is the relation between the areas and its perimeters of collapsed patches as $P \sim A^{H_{\mathrm{D}} / 2}$. We have found that while the power law exponent decreases as a function of the distribution factor $G / N$, the Hausdorff dimension decreases [Fig. 3(b)] following a power law relation $H_{\mathrm{D}}-1 \sim \varepsilon^{-3}$. This observation is a consequence of the spatial distribution: For $G / N=1$, the shape of the areas is compact, and the overlap (or the finite-size effect) is large. On the other hand, $G / N=0$ generates complex areas with large fractality and overlap which is much less probable, giving rise to the same power law exponents obtained by the single event model [Eq. (1)]. As a consequence of starting each avalanche on an empty frame, the occupation of the collapsed sites after each avalanche is small $(<10 \%)$ so that the critical percolation point is never reached.

We use the same procedure for the continuous events model where the model is written in terms of attempted and successful jerks:

$$
N_{a}(t)=f(t) N_{s}(t-1)+g(t) .
$$

In this model all collapse events take place in the same frame giving rise to a fully collapsed frame after a sufficiently long event time. We analyze the energy exponent as a function of time by taking the total number of all previous collapses produced after each time step. As previously, we distribute the collapsed sites by the $G / N$ probability. The events dataset is statistically poor for the initial short times and improves for longer times. For the case of $G / N=0$, the energy exponent of the jerks evolves towards $\varepsilon=1.67$ for long runs with high occupancies of the collapsed site. For $G / N=0.5$ we find $\varepsilon=$ 1.8 [Figs. 4(a) and 4(b)] for $80 \%$ collapsed sites.

After having explored the dynamical properties of the collapse model, we now investigate the topological features of the collapse patterns. The pattern evolution during avalanches was observed in the optical microscope in [34,57-60]. The most obvious parameter to characterize the evolving pattern is to determine the Hausdorff dimension of the collapsed sites as a function of time (or, equivalently, the fractal dimension). The Hausdorff dimension was measured by tracking the areas and perimeters of the collapsed areas at each time frame and the scaling $P \sim A^{H_{\mathrm{D}} / 2}[61]$ was used to determine $H_{\mathrm{D}}$ in Fig. 4. We then analyzed the distribution of the sizes of the collapsed areas as a function of time during the pattern formation. The total areas $A_{\text {tot }}$ for each time step are power law distributed with an exponent $\tau_{\text {topo }}$. The size of an avalanche $\mathrm{S}$ is traditionally associated with the transformed area $A_{\text {tot. }}$ In our model, the size and the amplitude $A_{\max }$ are proportional to each other so that one may be tempted to argue that the collapsed areas are a direct measure for the maximum amplitude of the avalanche so that it becomes important to understand the correlation between the two exponents $\tau_{\text {topo }}$ for $A_{\text {tot }}$ and $\tau^{\prime}$ for $A_{\max }$. Similarly, we define a new parameter as a topological equivalence of the "energy" of an avalanche as the square of the areas $\left(E_{\mathrm{topo}}=A_{\mathrm{total}}^{2}\right)$. The simulations show that this parameter is power law distributed with an exponent $\varepsilon_{\text {topo }}$ so that this exponent can be compared with the dynamical jerk energy exponent $\varepsilon$. The parameters describing the collapse pattern, namely, $\tau_{\text {topo }}$ and $\varepsilon_{\text {topo }}$, are directly correlated with the

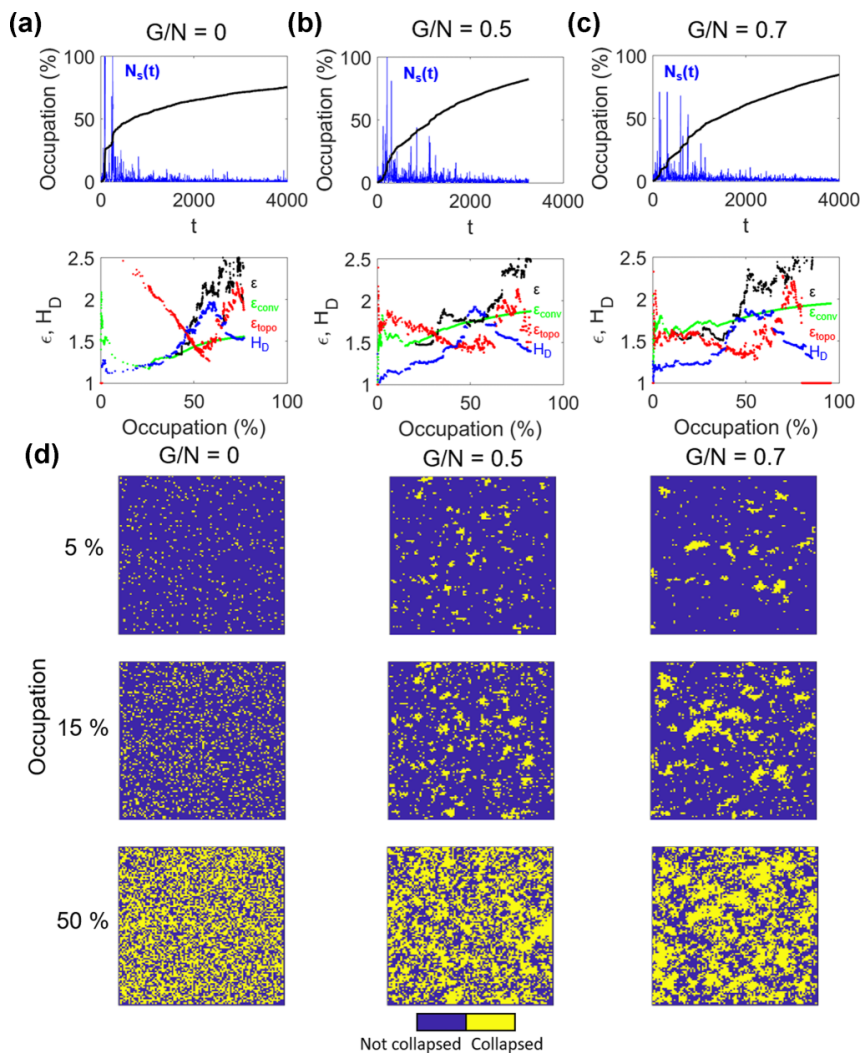

FIG. 4. Spatial pattern formation of the continuous model. $N_{s}(t)$ continuous avalanche profile (in blue) and its respective occupation (in black) for $G / N=0$, (a), 0.5 (b), and 0.7 (c). The lower panels show the time evolution of the Hausdorff dimension $H_{\mathrm{D}} \pm 0.05$ (in blue), the dynamical $\varepsilon_{\text {dyn }} \pm 0.05$, and the convergent $\varepsilon_{\text {conv }} \pm 0.05$ (errors for occupation larger than 20\%) power law exponent (black and green dots) extracted from the continuous profile $N_{s}(t)$ and the power law exponent obtained from the area's distribution $\varepsilon_{\text {topo }}$ (red dots) for $G / N=0$, (a), 0.5 (b), and 0.7 (c). (d) Images of the collapsed areas at different occupations for $G / N=0,0.5$, and 0.7 .

third topological descriptor, namely, the Hausdorff dimension $H_{\mathrm{D}}$ [Figs. 4(a)-4(c)].

The results shown in Fig. 4 show that the dominant avalanche activity occurs at times before 2000 time steps. After this time, the occupancy of the collapsed regions is around $60 \%$. After this degree of collapse, the avalanches become weak and cannot be followed on a reasonable computational timescale for occupancies beyond $80 \%$. For small occupancies, we find that $\varepsilon_{\text {topo }}$ and $H_{\mathrm{D}}$ are anticorrelated for all $G / N$ values. With increasing occupancy, the Hausdorff dimension increases from unity to a maximum near 2 . The maximum of $H_{\mathrm{D}}$ corresponds to a minimum of $\varepsilon_{\mathrm{top}}$. In particular, when the total collapsed sites occupy $50 \%$ of the available space, we find a maximum of $H_{\mathrm{D}}$ with $H_{\mathrm{D}}=1.95$ and a minimum of the topological energy exponent with $\varepsilon_{\text {topo }}=1.33$. The same exponent is expected from random-site $(G / N \ll 1)$ percolation in two dimensions at the critical point [56]. For larger values of $G / N$ it becomes difficult to define $H_{\mathrm{D}}$ and $\varepsilon_{\text {topo }}$ because the number of collapsed areas is small. In the extreme case for $G / N=1$ there is only one single collapsed area. Figure 4(d) 


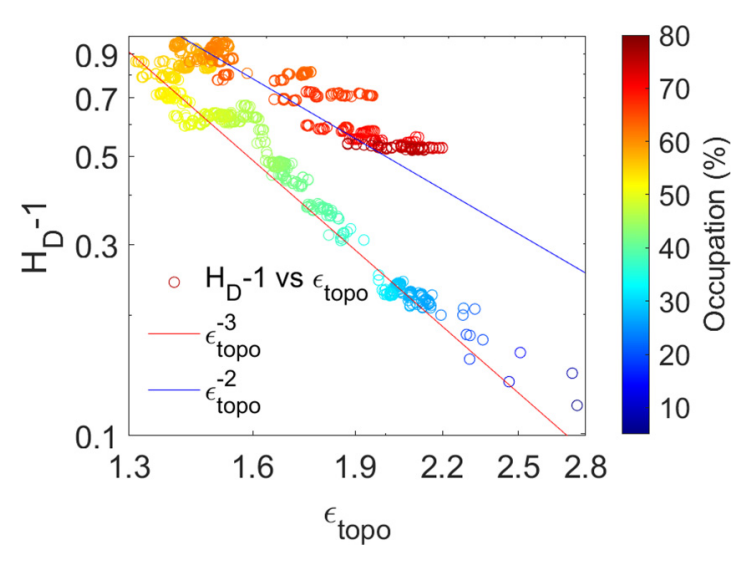

FIG. 5. $H_{\mathrm{D}}$ versus $\varepsilon_{\text {topo }}$ scaling for the continuous event model. $H_{\mathrm{D}} \pm 0.05$ is a function of the pattern formation exponent $\varepsilon_{\text {topo }} \pm$ 0.05 for $G / N=0$. The scaling depends on the occupation; below $50 \%$ follows $H_{\mathrm{D}}-1 \sim \varepsilon_{\text {topo }}^{-3}$ and above 50\%: $H_{\mathrm{D}}-1 \sim \varepsilon_{\text {topo }}^{-2}$. The exponents represent rough estimates in view of the large degree of data scatter. Nevertheless, the systematic anticorrelation between the Hausdorff dimension and the topological energy exponent is clearly visible.

shows snapshots of the collapsed areas as a function of the occupation and the $G / N$ factor.

The dynamical energy exponents $\varepsilon$, extracted from shifting time intervals by 300 time steps, deviate greatly from their topological equivalents. In the case of $G / N=0$ and for small occupancies, the dynamical $\varepsilon$ starts from the mean field value 1.33 and increases to values greater than 2 for occupancies beyond $50 \%$. The topological exponent $\varepsilon_{\text {topo }}$ starts from high values and then decays at its minimum values near 1.33. Despite these systematic differences, both exponents cover a similar parameter space between 1.33 and 2.5. At higher occupancies $\varepsilon_{\text {topo }}$ increases and becomes closer to the dynamical jerk exponent $\varepsilon$. The scaling of $\varepsilon_{\text {topo }}$ with the Hausdorff dimension is shown in Fig. 5 where a change of scaling is observed at around 50\% occupancy. For occupancies below $50 \%$, we notice a similar scaling as in the single event model when plotting $H_{\mathrm{D}}$ as a function of $\varepsilon$ for different values of $G / N$ [Fig. 3(b)]. We then calculate the energy exponent over increasing datasets $\varepsilon_{\text {conv }}$, starting from the beginning in which the dataset is statistically poor because few events occurred, which explains the scatter for occupancies below 10\%. The measured $\varepsilon_{\text {conv }}$ shows a minimum at 1.33 and converges for higher occupancies towards 1.66 for $G / N=0$ [Fig. 4(a)]. For higher values of $G / N$, the maximum of $\varepsilon_{\text {conv }}$ increases to values near 2 while the minimum increases to near 1.6 and coincides with $\varepsilon$ [Figs. 4(a)-4(c)]. In this scenario, if a large $\varepsilon_{\text {conv }}$ is measured in the full dataset it is related to a large $G / N$ factor.

The continuous event model is similar to the single event model at the dilute limit (for low occupancies). In this regime, the dynamic epsilon is constant as a function of the occupancy and increases for larger $G / N$ factors as a power law [as shown in Fig. 3(b)].

\section{CONCLUSIONS}

The models introduced in this paper are extremely simple and can be used to simulate avalanches on small computers without the need for mainframe computations. They reveal the main results of avalanches, as stimulated by the research in porous collapse and ferroic domain displacements under fields. These parameters are the exponents for energies, amplitudes, sizes, and so on. The unique aspect is that they combine the temporal correlations during avalanches and the resulting spatial pattern formation. Both aspects are key experimental quantities as measured by standard experimental techniques such as acoustic emission spectroscopy for time sequences of temporary jerks, and optical observations of the change of domain structures under applied fields. These parameters are naturally connected, and a first approach would be to identify a transformed region with a certain jerk amplitude or jerk energy. While this is approximately true it fails in detail. The two exponents $\varepsilon$ and $\varepsilon_{\text {topo }}$ are not identical and their dependence on the occupancy of the collapsed regions is different (Fig. 4). In the dilute limit, the dynamical exponent $\varepsilon$ is hardly influenced by the geometrical constraints ("growth"/"nucleation" $G / N$ factor). The topological exponent $\varepsilon_{\text {topo }}$ relates to the pattern formation process and includes the effect of the fractal structure of the collapsed regions. This fractality is also measured by the Hausdorff dimension and a clear anticorrelation is observed: A maximum in the exponent $\varepsilon_{\text {topo }}$ correlates with a minimum of the Hausdorff dimension. A similar anticorrelation was experimentally observed during ferroelectric switching in $\mathrm{BaTiO}_{3}$ [34].

\section{ACKNOWLEDGMENTS}

This research was supported by a grant from EPSRC (Grant No. EP/P024904/1). We thank Ignacio Pagonabarraga Mora and Raul Cruz Hidalgo for fruitful discussions about the model.
[1] B. Gutenberg and C. F. Richter, Nature (London) 156, 371 (1945).

[2] B. Gutenberg and C. F. Richter, Bull. Seismol. Soc. Am. 34, 185 (1944).

[3] Y. Ogata, J. Am. Stat. Assoc. 83, 9 (1988).

[4] Y. Y. Kagan and D. D. Jackson, Geophys. J. Int. 104, 117 (1991).

[5] R. Burridge, Geophys. J. Int. 35, 439 (1973).

[6] W. Klein, H. Gould, K. F. Tiampo, J. B. Silva, T. Gu, J. Kazemian, C. Serino, and J. B. Rundle, in Avalanches in
Functional Materials and Geophysics, edited by E. K. H. Salje, A. Saxena, and A. Planes (Springer, Berlin, 2017), pp. 1-18.

[7] D. L. Turcotte and S. R. Brown, Phys. Today 46(5), 68 (1993).

[8] F. Kun, Z. Halász, J. S. Andrade, Jr., and H. J. Herrmann, J. Stat. Mech. Theory Exp. (2009) P01021.

[9] S. Pradhan, A. Hansen, and P. C. Hemmer, Phys. Rev. Lett. 95, 125501 (2005).

[10] S. Pradhan and A. Hansen, Phys. Rev. E 72, 026111 (2005). 
[11] F. Raischel, F. Kun, and H. J. Herrmann, Phys. Rev. E 74, 035104 (2006).

[12] J. P. Sethna, K. A. Dahmen, and C. R. Myers, Nature (London) 410, 242 (2001).

[13] S. Zapperi, P. Cizeau, and G. Durin, Phys. Rev. B 58, 6353 (1998).

[14] D. Spasojević, S. Bukvić, S. Milošević, and H. E. Stanley, Phys. Rev. E 54, 2531 (1996).

[15] M. C. Gallardo, J. Manchado, F. J. Romero, J. del Cerro, E. K. H. Salje, A. Planes, E. Vives, R. Romero, and M. Stipcich, Phys. Rev. B 81, 174102 (2010).

[16] K. J. Måløy, S. Santucci, J. Schmittbuhl, and R. Toussaint, Phys. Rev. Lett. 96, 045501 (2006).

[17] D. Bonamy, S. Santucci, and L. Ponson, Phys. Rev. Lett. 101, 045501 (2008).

[18] J. Barés, A. Dubois, L. Hattali, D. Dalmas, and D. Bonamy, Nat. Commun. 9, 1253 (2018).

[19] K. E. Daniels and N. W. Hayman, J. Geophys. Res. Solid Earth 113, 11411 (2008).

[20] C. S. Campbell, Annu. Rev. Fluid Mech. 22, 57 (1990).

[21] M. Bouzid, A. Izzet, M. Trulsson, E. Clément, P. Claudin, and B. Andreotti, Eur. Phys. J. E 38, 125 (2015).

[22] J. Barés, D. Wang, D. Wang, T. Bertrand, C. S. O’Hern, and R. P. Behringer, Phys. Rev. E 96, 052902 (2017).

[23] X. Clotet, J. Ortín, and S. Santucci, Phys. Rev. E 93, 012149 (2016).

[24] R. Planet, S. Santucci, and J. Ortín, Phys. Rev. Lett. 102, 094502 (2009).

[25] R. Dickman, Phys. Rev. Lett. 90, 108701 (2003).

[26] O. Peters, C. Hertlein, and K. Christensen, Phys. Rev. Lett. 88, 018701 (2001).

[27] E. K. H. Salje, X. Ding, Z. Zhao, T. Lookman, and A. Saxena, Phys. Rev. B 83, 104109 (2011).

[28] X. Jiang, H. Liu, I. G. Main, and E. K. H. Salje, Phys. Rev. E 96, 023004 (2017).

[29] J. B. Mallinson, S. Shirai, S. K. Acharya, S. K. Bose, E. Galli, and S. A. Brown, Sci. Adv. 5, eaaw8438 (2019).

[30] F. Kun, I. Varga, S. Lennartz-Sassinek, and I. G. Main, Phys. Rev. E 88, 062207 (2013).

[31] F. Kun, I. Varga, S. Lennartz-Sassinek, and I. G. Main, Phys. Rev. Lett. 112, 065501 (2014).

[32] E. K. H. Salje, A. Planes, and E. Vives, Phys. Rev. E 96, 042122 (2017).

[33] Y. Chen, B. Gou, W. Fu, C. Chen, X. Ding, J. Sun, and E. K. H. Salje, Appl. Phys. Lett. 117, 262901 (2020).

[34] B. Casals, G. F. Nataf, and E. K. H. Salje, Nat. Commun. 12, 345 (2021).

[35] J. Baró, Á. Corral, X. Illa, A. Planes, E. K. H. Salje, W. Schranz, D. E. Soto-Parra, and E. Vives, Phys. Rev. Lett. 110, 088702 (2013).

[36] E. K. H. Salje, G. I. Lampronti, D. E. Soto-Parra, J. Baró, A. Planes, and E. Vives, Am. Mineral. 98, 609 (2013).
[37] G. F. Nataf, P. O. Castillo-Villa, J. Baró, X. Illa, E. Vives, A. Planes, and E. K. H. Salje, Phys. Rev. E 90, 022405 (2014).

[38] E. K. H. Salje, D. E. Soto-Parra, A. Planes, E. Vives, M. Reinecker, and W. Schranz, Philos. Mag. Lett. 91, 554 (2011).

[39] E. K. H. Salje, H. Liu, Y. Xiao, L. Jin, A. Planes, E. Vives, K. Xie, and X. Jiang, Phys. Rev. E 99, 023002 (2019).

[40] E. K. H. Salje and K. A. Dahmen, Annu. Rev. Condens. Matter Phys. 5, 233 (2014).

[41] E. K. H. Salje, D. Xue, X. Ding, K. A. Dahmen, and J. F. Scott, Phys. Rev. Mater. 3, 014415 (2019).

[42] C. D. Tan, C. Flannigan, J. Gardner, F. D. Morrison, E. K. H. Salje, and J. F. Scott, Phys. Rev. Mater. 3, 034402 (2019).

[43] See Supplemental Material at http://link.aps.org/supplemental/ 10.1103/PhysRevE.104.054138 for avalanche statistics for different Gaussian widths and the scaling relations for the continuous events model.

[44] B. Casals, K. A. Dahmen, B. Gou, S. Rooke, and E. K. H. Salje, Sci. Rep. 11, 5590 (2021).

[45] Y. Chen, Q. Wang, X. Ding, J. Sun, and E. K. H. Salje, Appl. Phys. Lett. 116, 111901 (2020).

[46] Y. Chen, X. Ding, D. Fang, J. Sun, and E. K. H. Salje, Sci. Rep. 9, 1330 (2019).

[47] D. Soto-Parra, X. Zhang, S. Cao, E. Vives, E. K. H. Salje, and A. Planes, Phys. Rev. E 91, 060401 (2015).

[48] X. He, X. Ding, J. Sun, and E. K. H. Salje, Appl. Phys. Lett. 108, 072904 (2016).

[49] A. P. Mehta, K. A. Dahmen, and Y. Ben-Zion, Phys. Rev. E 73, 056104 (2006).

[50] L. Laurson, X. Illa, S. Santucci, K. Tore Tallakstad, K. J. Måløy, and M. J. Alava, Nat. Commun. 4, 2927 (2013).

[51] E. K. H. Salje, H. Liu, L. Jin, D. Jiang, Y. Xiao, and X. Jiang, Appl. Phys. Lett. 112, 054101 (2018).

[52] T. Utsu and Y. Ogata, J. Phys. Earth 43, 1 (1995).

[53] X. Jiang, D. Jiang, J. Chen, and E. K. H. Salje, Am. Mineral. 101, 2751 (2016).

[54] E. W. Carlson, S. Liu, B. Phillabaum, and K. A. Dahmen, J. Supercond. Novel Magn. 28, 1237 (2015).

[55] B. Phillabaum, E. W. Carlson, and K. A. Dahmen, Nat. Commun. 3, 915 (2012).

[56] M. Corsten, N. Jan, and R. Jerrard, Phys. A (Amsterdam, Neth.) 156, 781 (1989).

[57] B. Casals, S. van Dijken, G. Herranz, and E. K. H. Salje, Phys. Rev. Research 1, 032025(R) (2019).

[58] B. Casals, G. F. Nataf, D. Pesquera, and E. K. H. Salje, APL Mater. 8, 011105 (2020).

[59] R. Niemann, J. Kopeček, O. Heczko, J. Romberg, L. Schultz, S. Fähler, E. Vives, L. Mañosa, and A. Planes, Phys. Rev. B 89, 214118 (2014).

[60] X. Balandraud, N. Barrera, P. Biscari, M. Grédiac, and G. Zanzotto, Phys. Rev. B 91, 174111 (2015).

[61] G. Catalan, H. Béa, S. Fusil, M. Bibes, P. Paruch, A. Barthélémy, and J. F. Scott, Phys. Rev. Lett. 100, 027602 (2008). 